\title{
Molecular characterization of grapevine yellow speckle viroid-2 (GYSVd-2)
}

\author{
Dongmei Jiang $\cdot$ Zhixiang Zhang $\cdot$ Zujian Wu $\cdot$ \\ Rui Guo $\cdot$ Hongqing Wang $\cdot$ Peige Fan $\cdot$ Shifang Li
}

Received: 31 December 2008/ Accepted: 12 February 2009/Published online: 3 March 2009

(C) The Author(s) 2009. This article is published with open access at Springerlink.com

\begin{abstract}
Grapevine yellow speckle viroid-2 (GYSVd-2) is a viroid found only in grapevines in China and Australia. Here, we report the molecular characterization of GYSVd2 isolated from three grapevine varieties in China. A total of $90 \mathrm{cDNA}$ clones were sequenced including $30 \mathrm{cDNA}$ clones obtained from each of the Black Olympia, Zaoyu, and Thomson Seedless isolates. Sequencing analysis identified 20, 18, and 12 different sequence variants from the 3 isolates, respectively. Furthermore, each of the isolates included one predominant sequence variant. Compared to the Australian variant of GYSVd-2 (Accession number: NC_003612), the Black Olympia variant was identical and the Zaoyu variant contained one substitution. In contrast, the Thomson Seedless isolate significantly varied from the Australian variant with three substitutions, two insertions, and four deletions. In silico structure
\end{abstract}

D. Jiang $\cdot$ Z. Zhang $\cdot$ R. Guo $\cdot$ S. Li $(\bowtie)$

State Key Laboratory of Biology of Plant Diseases and Insect Pests, Institute of Plant Protection, Chinese Academy of Agricultural Sciences, Yuanmingyuan West Road No. 2, Haidian District, Beijing 100193, People's Republic of China

e-mail: sfli@ippcaas.cn; lishifang2003@yahoo.com.cn

D. Jiang $\cdot \mathrm{Z}$. Wu

Institute of Plant Virology, Fujian Agriculture and Forestry University, Jinshan, Fuzhou, Fujian 350002, People's Republic of China

H. Wang $(\bowtie)$

Department of Fruit Science, College of Agronomy and

Biotechnology, China Agricultural University, Beijing 100193,

People's Republic of China

e-mail: wanghq@cau.edu.cn

P. Fan

Institute of Botany, Chinese Academy of Sciences, Beijing

100093, People's Republic of China analysis predicted that the variations were clustered in the terminal left, the pathogenicity, and the variable region of the predicted secondary structure of GYSVd-2.

Keywords Grapevine yellow speckle viroid-2 - Genetic variation $\cdot$ Sequencing analysis $\cdot$ Secondary structure

\section{Introduction}

Viroids are small, circular, single-stranded RNA molecules that range in size from 246 to 475 nucleotides (nts). Viroids replicate in host plants and act as phytopathogenic agents; however, unlike viruses, viroids do not code for proteins. Viroids are the smallest known plant pathogens and are responsible for several economically significant crop diseases [1]. Viroids are classified into two families: Pospiviroidae, which is composed of species with a central conserved region (CCR) that do not contain a hammerhead ribozyme, and Avsunviroidae, a family composed of species lacking a CCR but containing a hammerhead ribozyme that is able to self-cleave both strands of the viroid [2].

Up to now, five viroids have been identified with the ability to infect grapevines: Hop stunt viroid (HSVd) [3], Citrus exocortis viroid (CEVd) [4], Australian grapevine viroid (AGVd) [5], and two Grapevine yellow speckle viroids (GYSVd-1 and GYSVd-2) [6, 7]. Grapevine viroids have been divided into three genera based on sequence homology of their CCRs [8]. GYSVd-1, GYSVd-2, and AGVd are classified in the Apscaviroid group, whereas HSVd-g [9] and CEVd-g are classified in the Hostuviroid and Pospiviroid groups, respectively [10]. Mixed infections involving these viroids in cultivated grapevines is common, and in general, grapevine viroids produce very few, if any, disease symptoms, thereby allowing these viroids to 
replicate in a host unnoticed. However, of the five types of grapevine viroids identified, GYSVd-1 and GYSVd-2 are associated with disease symptoms such as yellow speckle, which was originally identified under hot greenhouse conditions in Australia [6, 7, 11].

GYSVd-2 is composed of 363 nts and was first detected in Australia when the grapevine cultivar 'Kyoto' was grafted onto the 'Dogridge' rootstock and subsequently became infected with yellow speckle disease [6]. GYSVd-2 is the most closely related viroid to GYSVd-1 with an overall sequence similarity of $73 \%$, which accounts for the cross-hybridization that occurs between the two species [7]. Although GYSVd-2 RNA can be separated from other grapevine viroid RNA of similar size, including GYSVd-1, separation occurs only after prolonged electrophoresis under denaturing conditions [7]. Until now, GYSVd-2 has only been identified in grapevines from Australia and China [6, 12].

Due to the low infection rate of cultivated grapevines by GYSVd-2, little is known about this viroid. The present work characterizes GYSVd-2 isolates collected from three different grapevine varieties in China to provide new information regarding the population diversity and the genetic variation of the viroid. Differences in viroid sequences identified among isolates from grapevines in China as well as in comparison with an Australian GYSVd2 isolate indicate a novel isolate has been identified in Xinjiang, China, distinct from the other isolates collected from Beijing, China, and Australia.

\section{Materials and methods}

\section{Viroid sources}

From 2006 to 2008, young leaves of 89 grapevine samples from different grape varieties were collected from Xinjiang autonomous region, Shenyang, Fujian, and Beijing, China. They were detected by dot-blot or Northern hybridization using DIG-labeled GYSVd-1 riboprobe followed by RT-PCR using GYSVd-2 specific primers.

\section{Isolation and extraction of GYSVd-2}

Low molecular weight RNAs were extracted according to $\mathrm{Li}$ et al. [13]. Briefly, $5 \mathrm{~g}$ of tissue were treated with liquid nitrogen, extracted with $10 \mathrm{ml}$ of $1 \mathrm{M} \mathrm{K}_{2} \mathrm{HPO}_{4}$ containing $0.1 \% \beta$-mercaptoethanol, and homogenized with $10 \mathrm{ml}$ phenol:chloroform $(1: 1, \mathrm{v} / \mathrm{v})$. To eliminate polysaccharides present, extraction with 2-methoxyethanol and Cetyltrimethyl Ammonium Bromide (CTAB) precipitation were performed, followed by treatment with $2 \mathrm{M} \mathrm{LiCl}$ to isolate the soluble fraction and collect the low molecular weight RNAs. The resulting preparation was dissolved in $30 \mu \mathrm{l}$ of distilled water.

Reverse transcription-polymerase chain reaction (RTPCR)

Using RT-PCR, cDNAs were generated from viroid RNA. Briefly, template $(1 \mu \mathrm{l})$ was mixed with $0.5 \mu \mathrm{l}(20 \mathrm{pmol})$ of primer GYSVd-2-P2 (5'-ACTAGTCCGAGGACCTTTTC TAGCGCTC-3', complementary to nucleotides 166-187) and distilled water, then heated at $98^{\circ} \mathrm{C}$ for $5 \mathrm{~min}$, and quenched in ice water for more than $2 \mathrm{~min}$. Then $1 \mu \mathrm{l}$ of $2.5 \mathrm{mM}$ dNTPs, $1 \mu \mathrm{l}(200 \mathrm{U}) \mathrm{MMuLV}$ reverse transcriptase (Promega), $2 \mu \mathrm{l}$ M-MLV $5 \times$ reaction buffer, $0.25 \mu \mathrm{l}$ (40 U) Recombinant RNasin ribonuclease inhibitor, and distilled water were added to the RT mixture for a final volume of $10 \mu \mathrm{l}$. The resulting mixture was incubated at $42^{\circ} \mathrm{C}$ for $60 \mathrm{~min}$, then at $98^{\circ} \mathrm{C}$ for $5 \mathrm{~min}$. After the RT reaction, $5 \mu \mathrm{l}$ of the reverse transcription solution was mixed with $25 \mu \mathrm{l} 2 \times$ PCR Ex-Taq Mix, $18 \mu$ l distilled water, and $1 \mu \mathrm{l}(20 \mathrm{pmol})$ each of primers GYSVd-2-P1 (5'-ACTAGTACTTTCTTCT ATCTCCGAAGC-3' ${ }^{\prime}$, homologous to nucleotides 188-208) and GYSVd-2-P2 (5'-ACTAGTCCGAGGACCTTTTCT AGCGCTC- $3^{\prime}$ ) to yield a final reaction volume of $50 \mu \mathrm{l}$. The cycling parameters for PCR amplification consisted of one cycle of heat denaturation at $94^{\circ} \mathrm{C}$ for $5 \mathrm{~min}, 30$ amplification cycles of $94^{\circ} \mathrm{C}$ for $30 \mathrm{~s}, 56^{\circ} \mathrm{C}$ for $30 \mathrm{~s}$, and $72^{\circ} \mathrm{C}$ for $30 \mathrm{~s}$, and a final elongation step at $72^{\circ} \mathrm{C}$ for $5 \mathrm{~min}$.

Cloning and sequencing

After RT-PCR, electrophoresis confirmed the presence of the expected PCR products before they were purified using a PCR purification kit (Tiangen). The resulting fragments were cloned into the pMD18-T vector (Takara) and transformed into $E$. coli DH5 $\alpha$. Recombinant DNA clones containing a 363-bp insert were identified by restriction analysis. Selected clones were sequenced using an automated DNA sequencer (ABI PRISMTM 3730XL DNA Analyzer) and analyzed by DNAMAN Version 5.2.2.

Sequencing analysis and determination of secondary structures

Sequences were aligned with the Australian GYSVd-2 sequence deposited in the GenBank database (Accession number: NC_003612) using Clustal W (Ver.1.83). Possible secondary structures were calculated using the CLC RNA Workbench package (Version 3.0.1, http://www.clcrna workbench.com/). 


\section{Results}

Genetic diversity of GYSVd-2 within each isolate of Black Olympia, Zaoyu, and thomson seedless

GYSVd-2 was positive in only three samples of the 89 grapevine samples examined, i.e., one each from the cultivars Black Olympia, Zaoyu, and Thomson Seedless. The Black Olympia and Zaoyu samples were collected from the same grapevine nursery in Beijing, while the Thomson Seedless sample was collected from a grapevine tree from the Xinjiang autonomous region estimated to be older than 150 years. Thirty cDNA clones were chosen randomly from each of the three isolates, and a total of 90 independent cDNA clones were sequenced. Of them, a total of 50 sequence variants were identified. The variants detected in the Black Olympia isolate included one predominant sequence variant, Bv1 (363 nts) and 19 singletons (Fig. 1, left). In the Zaoyu isolate, one predominant sequence variant, Zv1 (363 nts) was identified along with 17 singletons (Fig. 1, middle). In the Thomson Seedless isolates, one predominant sequence variant, Tv1 (361 nts) was detected along with 11 singletons (Fig. 1, right). Each of the three isolates consisted of one predominant sequence variant which occupied $37 \%, 43 \%$, or $63 \%$ of the sequences determined in each isolate (Fig. 1). The overall sequence homology within each isolate was high, for example, the homology between the 20 sequence variants of Black Olympia isolate was $98.90-99.72 \%$. Based on the identification of one predominant sequence variant in each of the isolates (i.e., Bv1, Zv1, and Tv1), we hypothesize that all the three GYSVd-2 isolates form a quasispecies with one predominant sequence [11, 12, 14-17].

Genetic diversity among GYSVd-2 isolates from different grapevine varieties

When the sequences of Bv1, Zv1, and Tv1 were aligned with the GYSVd-2 sequence derived from an Australian grapevine (Accession number: NC_003612), zero, one, and nine positions showed differences, resulting in an overall sequence homology of $100 \%, 99.72 \%$, and $98.34 \%$, respectively, in each case. The high degree of homology for the Black Olympia and Zaoyu predominant variants, except at position $300\left(A_{300} \rightarrow G\right)$ for $Z v 1$, was consistent with the origination of the two isolates from the same nursery yard in Beijing. We hypothesize that Bv1 and Zv1 have the same origin and were geographically separated rather recently. In contrast, the predominant sequence of the Thomson Seedless isolate, Tv1, differed from the Australian isolate with three substitutions at positions 300 $\left(\mathrm{A}_{300} \rightarrow \mathrm{G}\right), 361\left(\mathrm{C}_{361} \rightarrow \mathrm{T}\right)$, and $362\left(\mathrm{~T}_{362} \rightarrow \mathrm{C}\right)$, along with two insertions at positions $134\left(-{ }_{134} \rightarrow \mathrm{A}\right)$ and 147 $\left({ }_{147} \rightarrow \mathrm{T}\right)$, and four deletions at positions $15\left(\mathrm{~T}_{15} \rightarrow-\right)$, $349\left(\mathrm{C}_{349} \rightarrow-\right), 350\left(\mathrm{G}_{350} \rightarrow-\right)$, and $357\left(\mathrm{G}_{357} \rightarrow-\right)$ (Fig. 2). The substitution at position $300\left(A_{300} \rightarrow G\right)$ is the same as the one identified in the Zaoyu isolate. Given the greater number of differences identified in Tv1 versus the Australian sequence variant, $\mathrm{Bv} 1$ and $\mathrm{Zv} 1$, it appears that $\mathrm{Tv} 1$ is a more isolated viroid variant, consistent with its collection from a 150-year-old grapevine tree growing in Xinjiang.

Effects of genetic diversity on predicted secondary structures

Most of the variations found in the three GYSVd-2 isolates from China were clustered in the terminal left (TL) region, the pathogenicity-associated region $(\mathrm{P})$, and the variable (V) region of the viroid-predicted secondary structure. Variations were not found in the CCR (from 84-120 nts) or in the terminal conserved region (TCR) of the TL region (Fig. 3). In silico analysis of the predicted secondary structures of the variants suggested that some of the sequence variants could influence the viroid structure. Specifically, the two mutations at positions 134 and 147 of the Thomson Seedless (Tv1) variant were predicted to
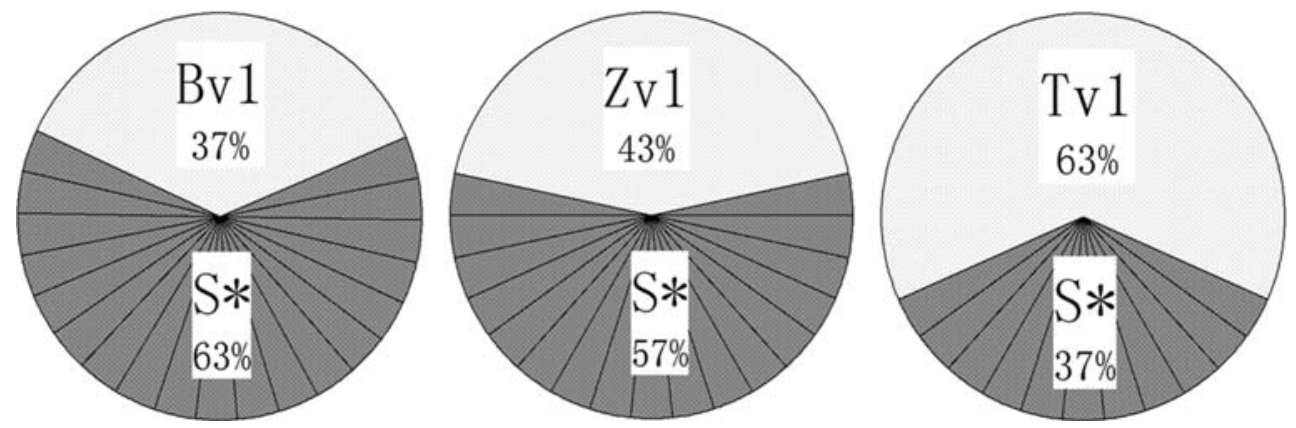

Fig. 1 GYSVd-2 variants from the three different grape varieties (Black Olympia, Zaoyu, and Thomson Seedless) in China. Thirty each of independent cDNA clones, respectively, from each of the three isolates, in total of 90 , were sequenced. One predominant sequence was identified for each: Bv1 for Black Olympia, Zv1 for Zaoyu, and Tv1 for Thomson Seedless. The percentage of variants associated with singletons $\left(\mathrm{S}^{*}\right)$ are also indicated in dark gray 
Fig. 2 Sequence alignment of GYSVd-2 variants isolated from grapevines in China and Australia. Sequences of viroids isolated from different grapevines in China versus a previously published GYSVd-2 sequence from a grapevine in Australia (Accession number: NC_003612). The position mutations of specific sequence variants are boxed and the CCR domain is labeled above the sequence. (AUS: Australia, Bv1: China (Black Olympia), Zv1: China (Zaoyu), Tv1: China (Thomson Seedless))
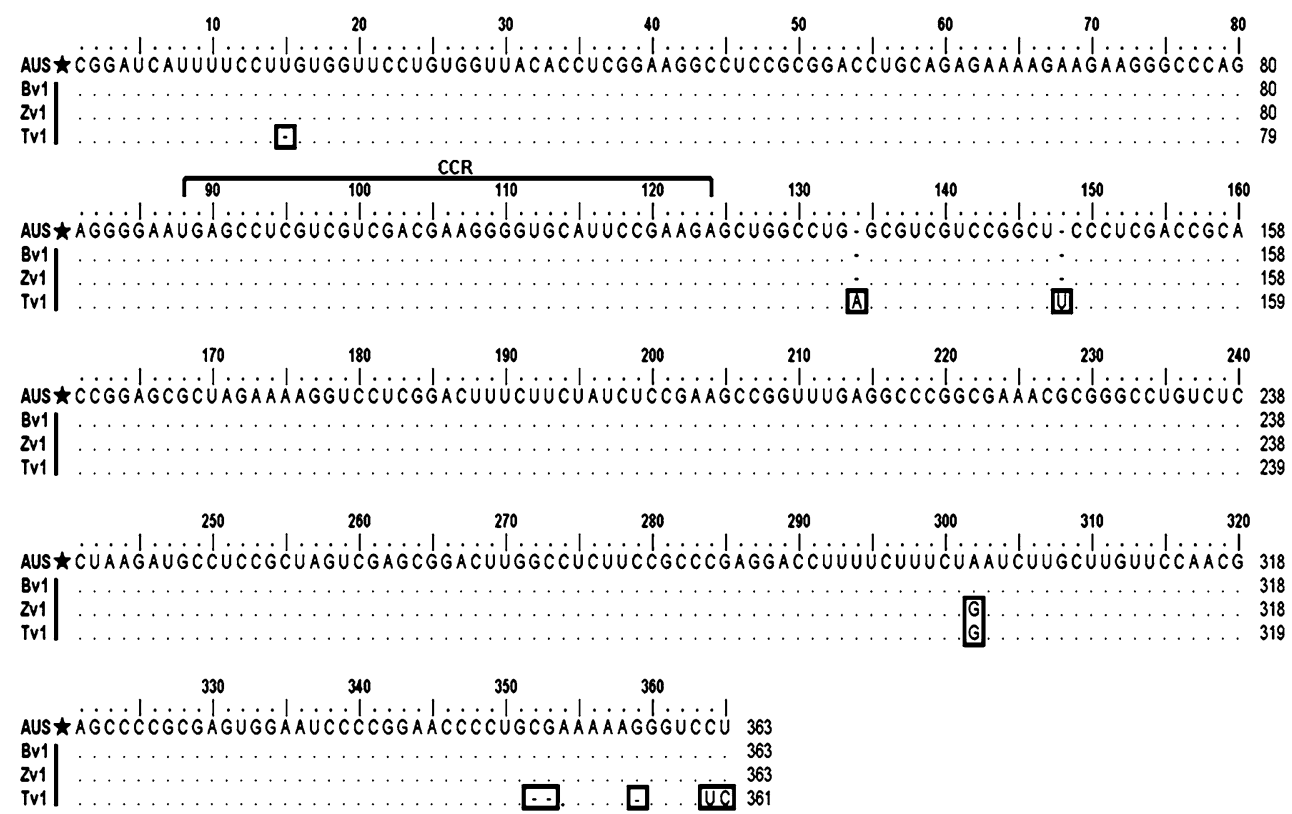

affect the predicted secondary structure (Fig. 3). In silico structure analysis also revealed that the $\mathrm{P}$ domain of GYSVd-2 is purine rich in the upper strand and uridine rich in the bottom strand.

\section{Discussion}

Here, we present, for the first time, the population diversity and genetic variations of the viroid, GYSVd-2, identified from three isolates (Black Olympia, Zaoyu, and Thomson Seedless) collected from grapevines in China. In total of 90 cDNA clones, 30 each of independent cDNA clones from the 3 isolates were sequenced. Twenty sequence variants from Black Olympia, 18 sequence variants from Zaoyu, and 12 sequence variants from Thomson Seedless, in total of 50, were identified. Although it is possible that the singletons identified could represent PCR artifacts, we hypothesize that they are naturally occurring mutations for the following reasons. First, the frequency of error resulting from Ex-Taq DNA polymerase ranges from $10^{-4}$ to $10^{-5}$ [18]. Second, most of the mutations were identified multiple times in different cDNA clones. For example, all the sequence variants showed an $A$ to $G$ substitution at nucleotide 300 in both the Thomson Seedless and the Australian isolates [7]. Additionally, most of the single mutation sites were located in the TL, P, and V domains of GYSVd-2, and not in the strictly conserved regions of the CCR and the TCR of Apscaviroid, supporting the hypothesis that these mutations are naturally occurring.

Sequence alignments between the variants isolated from grapevines in Australia and China demonstrate that GYSVd-2 isolates from different countries do not necessarily display regional disparity and variety-specific sequence variants. For example, Bv1, the predominant sequence of Black Olympia, was identical to the Australian sequence and represented the first reported variant of the Australian isolate. Since Black Olympia and Zaoyu isolates were collected from the same grapevine nursery in Beijing, and the homology between the Black Olympia, Zaoyu, and the Australian isolate were similar, it appears that they derived from a common ancestor. In contrast, sequence variations were found at nine different positions in the Thomson Seedless isolate versus the other grapevine varieties. Interestingly, six out of the nine single site mutations were located in the TL region (Fig. 3). This is in contrast with previous studies that have found the TCR of Apscaviroid species (CNNGNGGUUCCUGUGG) to be highly conserved in the TL region [19-21]. This difference may be explained by the fact that the Thomson Seedless isolate was collected from a grapevine tree estimated to be older than 150 years, which is predicted to have survived by its own root given the absence of any records of grafting or top-working manipulations. Therefore, the increased number of mutations in a region otherwise found to be highly conserved is consistent with the extended period of isolation experienced by this grapevine tree that grows in a latitude and climate distinct from the other grapevine varieties analyzed.

The most stable secondary structures for the three predominant variants (Bv1, Zv1, and Tv1) were derived based on energy calculations. We also reconfirmed that the $\mathrm{P}$ domain of GYSVd-2 is purine rich in the upper strand and uridine rich in the bottom strand, resulting in a relatively high sequence homology between the $\mathrm{P}$ domain of GYSVd-2 and the P domains of other viroids [8]. Like 


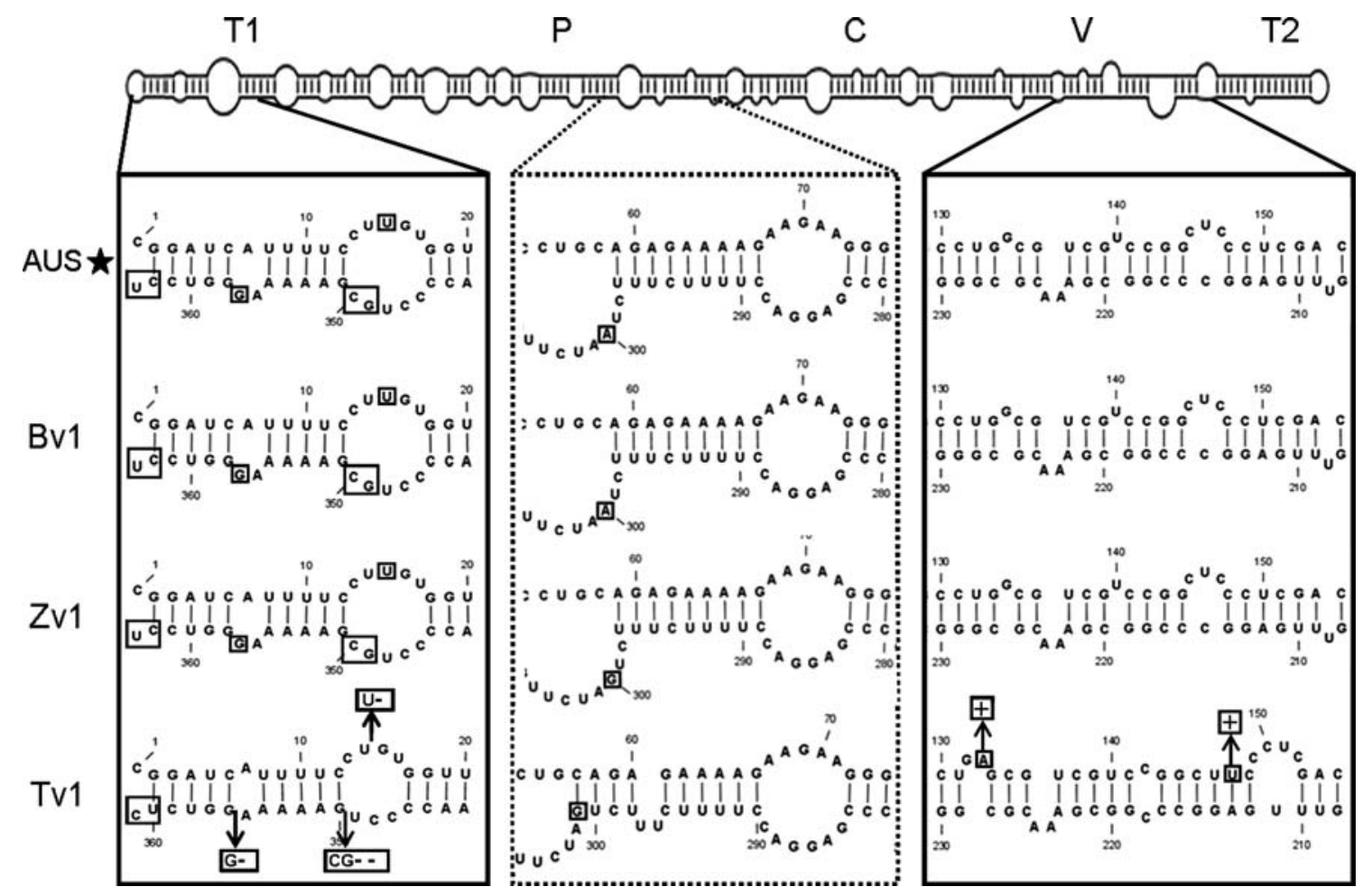

Fig. 3 Predicted secondary structures of GYSVd-2 isolates. Compared to the first reported sequence of GYSVd-2 from a grapevine in Australia (AUS) (Accession number: NC_003612), of which the Black Olympia (Bv1) isolate was identical, base changes in the Zaoyu (Zv1) and Thomson Seedless (Tv1) isolates are boxed. Insertions

other RNAs, the predicted secondary structure of GYSVd-2 contains many loops and bulges flanked by double-stranded helices, the biological functions of which are mostly unknown. Recent studies have demonstrated that the loops/ bulges in the rod-shaped secondary structure of the viroid serve as major functional motifs that likely interact with cellular factors to accomplish various aspects of replication and systemic trafficking during infection [22]. Compared to the Australian variant, most of the variations found in the Chinese isolates were located in the TL and P domains of the secondary structure, which may have some influence on the secondary structure of the GYSVd-2 viroid, particularly with respect to replication and pathogenicity of the viroid. However, these possibilities would require further investigation. Among the nucleotide positions that did exhibit substitutions, the $A_{300} \rightarrow G$ substitution in $\mathrm{Zv1}$ was not predicted to influence secondary structure, although when other base changes were present in Tv1, changes in the secondary structure were predicted (Fig. 4). We hypothesize that this might be due to reciprocity among the bases.

Characterization of the genetic structure and variation of a viroid population is important to understand the dissemination and evolution of a viroid family. With the advent of molecular virology, many viroid populations present in the Tv1 isolate are indicated with arrows and the inserted nucleotides are boxed. Additionally, mutations in Tv1 that affected secondary structure are indicated with boxed plus signs. (T1, T2: the terminal regions; $\mathrm{P}$ : the pathogenicity region; $\mathrm{C}$ : the central conserved region; $\mathrm{V}$ : the variable region)

Fig. 4 Analysis of the terminal conserved region (TCR) of GYSVd-2 isolates in the Apscaviroid group. The sequence proximal to the $3^{\prime}$ end of the motif is highly conserved while considerable variability is found in the nucleotides near the $5^{\prime}$ terminus as evidenced in the three "N" nts in the five terminal positions [22]. Sequences from viroids isolated from different grapevines in China are compared with a previously published GYSVd-2 sequence from a grapevine in Australia (AUS*) (Accession number: NC_003612) and a previously published ASSVd sequence (ASSVd) (Accession number: NC 001340). (Abbreviation of the sample names such as "Bv1" was described in the text. Samples with asterisk $(\star)$ indicate those obtained from GenBank.)

have been characterized including AFCVd, HSVd, CEVd, GYSVd-1, and AGVd [15, 16, 19, 23, 24, 25-27, 28, 29]. Given the limited distribution of GYSVd-2 in the world, very little is known about GYSVd-2 at the molecular level prior to this study. For example, GYSVd-2 was previously detected in 11 out of 27 grapevines characterized from 
Australia [30]. However, GYSVd-2 varieties present in China were not previously reported [12]. Recently, an intensive survey in Japan of 111 different grapevine cultivars in three different nursery yards and eight cultivars in several commercial vine yards have been investigated, however, GYSVd-2 has not been detected (T. Sano, personal communication).

An unusually high GYSVd-2 infection rate in Australian grapevines, combined with the unique sequence variations identified in this study of GYSVd-2 Xinjiang isolates, provide additional information regarding the biology of GYSVd-2. Insights into the GYSVd-2 viroid can also provide a possible model for the biology of other grapevine viroids which have expanded their distribution among the world viticulture. As a result, more extensive surveys on grapevine viroids present in Xinjiang, China, are now underway.

\section{GenBank accession numbers}

GenBank accession numbers for the sequence variants identified for GYSVd-2 isolated from China are FJ490172FJ490175 and FJ597915-FJ597947.

Acknowledgments This work was supported by grants from the National Basic Research and Development Program of China (973 Program) (No. 2009CB119200 and No. 2006 CB100203), the National Natural Science Foundation of China (No.30771403), the Beijing Natural Science Foundation of China (No. 6072022), the National Key Technologies Research and Development Program (No. 2006 BAD08A14), the National High Technology Research and Development Program (863 Program) (No. 2006AA10Z432) and the Opening Project of State Key Laboratory for Biology of Plant Diseases and Insect Pests, Institute of Plant Protection, Chinese Academy of Agricultural Sciences. This work was also supported by NSFC-JSPS Joint Research Project between China and Japan (30811140157). We specially thank Prof. Teruo Sano at Hirosaki University of Japan for his valuable comments and the critical reading of this manuscript.

Open Access This article is distributed under the terms of the Creative Commons Attribution Noncommercial License which permits any noncommercial use, distribution, and reproduction in any medium, provided the original author(s) and source are credited.

\section{References}

1. C.M. Fauquet, M.A. Mayo, J. Maniloff, U. Desselberger, L.A. Ball (eds.), in Virus Taxonomy. Eighth Report of the International Committee on Taxonomy of Viruses (Elsevier, 2005), pp. 1145-1160

2. A.M. Koltnuow, M.A. Rezaian, Intervirology 30(4), 194-201 (1989)
3. T. Sano, K. Ohshima, T. Hataya, I. Uyeda, E. Shikata, T.G. Chou, T. Meshi, Y. Okada, J. Gen. Virol. 67, 1673-1678 (1986). doi: 10.1099/0022-1317-67-8-1673

4. F. Garcia-Arenal, V. Pallas, R. Flores, Nucleic Acids Res. 12, 4203-4209 (1987). doi:10.1093/nar/15.10.4203

5. M.A. Rezaian, Nucleic Acids Res. 18, 1813-1818 (1990). doi: 10.1093/nar/18.7.1813

6. A.M. Koltunow, M.A. Rezaian, Nucleic Acids Res. 16, 849-864 (1988). doi:10.1093/nar/16.3.849

7. A.M. Koltunow, M.A. Rezaian, Virology 170, 575-578 (1989). doi:10.1016/0042-6822(89)90450-9

8. P. Keese, R.H. Symons, Proc. Natl. Acad. Sci. USA 82, 4582 4586 (1985). doi:10.1073/pnas.82.14.4582

9. T. Sano, K. Ohshima, T. Hataya, I. Uyeda, E. Shikata, T. Chou, T. Meshi, Y. Okada, J. Gen. Virol. 66, 333-338 (1985). doi: 10.1099/0022-1317-66-2-333

10. S.F. Elena, J. Dopazo, R. Flores, T.O. Diener, A. Moya, Proc. Natl. Acad. Sci. USA 88, 5631-5634 (1991). doi:10.1073/pnas. 88.13 .5631

11. R.H. Taylor, R.C. Woodham, Aust. J. Agric. Res. 23, 447-452 (1972). doi:10.1071/AR9720447

12. S.F. Li, R. Guo, S. Peng, T. Sano, Plant Pathol. 89, 572 (2007)

13. S.F. Li, S. Onodera, T. Sano, K. Yoshida, G.P. Wang, E. Shikata, Ann. Phytopathol. Soc. Jpn. 61, 381-390 (1995)

14. F.M. Codoner, J.A. Daros, R.V. Sole, S.F. Elena, PLoS Pathog. 2(12), e136 (2006). doi:10.1371/journal.ppat.0020136

15. T. Sano, S. Isono, K. Matsuki, Y. Kawaguchi-Ito, K. Tanaka, K. Kondo, A. Iijima, M. Bar-Joseph, Virus Genes 37, 298-303 (2008). doi:10.1007/s11262-008-0270-9

16. D.M. Jiang, S. Peng, Z.J. Wu, Z.M. Cheng, S.F. Li, Virus Genes 38, 178-183 (2009). doi:10.1007/s11262-008-0306-1

17. S. Ambros, C. Hernandez, J.C. Desvignes, R. Flores, J. Virol. 72, 7397-7406 (1998)

18. M.A. Bracho, E. Barrio, J. Gen. Virol. 79, 2921-2928 (1998)

19. H. Polivka, U. Staub, H.J. Gross, J. Gen. Virol. 77, 155-161 (1996). doi:10.1099/0022-1317-77-1-155

20. A.J. Dingley, G. Steger, B. Esters, D. Riesner, S. Grzesiek, J. Mol. Biol. 334, 751-767 (2003). doi:10.1016/j.jmb.2003.10.015

21. J.S. Semancik, G. Vidalakis, Arch. Virol. 150, 1059 (2005). doi: 10.1007/s00705-005-0499-8

22. X.H. Zhong, A.J. Archual, A.A. Amin, B. Ding, Plant Cell 20, 35-47 (2008). doi:10.1105/tpc.107.056606

23. A. Palacio-Bielsa, J. Romero-Durban, N. Duran-Vila, Arch. Virol. 149, 537-552 (2004). doi:10.1007/s00705-003-0223-5

24. T. Sano, R. Mimura, K. Ohshima, Virus Genes 22, 53 (2001). doi: 10.1023/A: 1008182302704

25. M. Gandia, L. Rubio, A. Palacio, N. Duran-Vila, Arch. Virol. 150, 1945-1957 (2005). doi:10.1007/s00705-005-0570-5

26. X. Foissac, N. Duran-Vila, Arch. Virol. 145, 1975-1983 (2000). doi:10.1007/s007050070070

27. J.E. Visvader, R.H. Symons, Nucleic Acids Res. 13, 2907-2920 (1985). doi:10.1093/nar/13.8.2907

28. J.A. Szychowski, R. Credi, K. Reanwarakorn, J.S. Semancik, Virology 248, 432-444 (1998). doi:10.1006/viro.1998.9292

29. A. Elleuch, H. Fakhfakh, M. Pelchat, P. Landry, M. Marrakchi, J.P. Perreault, Eur. J. Plant Pathol. 108, 815-820 (2002). doi: 10.1023/A:1020855405948

30. A.M. Koltunow, L.R. Krake, S.D. Johnson, M.A. Rezaian, J. Gen. Virol. 70, 3411-3419 (1989). doi:10.1099/0022-1317-70-12-3411 